\title{
LA EXTENSIÓN UNIVERSITARIA COMO CONSTRUCCIÓN DE LO COMÚN: UNA MIRADA PSICOSOCIAL DE LA INTERVENCIÓN COMUNITARIA EN LA LOCALIDAD DE LA SERRANITA, CÓRDOBA, ARGENTINA
}

\author{
A EXTENSÃO UNIVERSITÁRIA COMO CONSTRUÇÃO \\ DO COMUM: UMA VISÃO PSICOSSOCIAL DA \\ INTERVENÇÃO COMUNITÁRIA NA LOCALIDADE \\ DE SERRANITA, CÓRDOBA, ARGENTINA
}

\section{UNIVERSITY EXTENSION AS A CONSTRUCTION OF THE 'COMMUNAL': A PSICOSSOCIAL VIEW OF INTERVENTION IN THE COMMUNITY OF SERRANITA, CORDOBA, ARGENTINA}

Mariel Castagno* María Sol Díaz

\section{RESUMEN}

El presente trabajo retoma las acciones y reflexiones realizadas en el marco de una intervención extensionista en la localidad de La Serranita, Provincia de Córdoba, Argentina. Una experiencia sostenida a lo largo de tres años que tuvo como origen primigenio un curso de extensión "Clínica Social: Herramientas prácticas en Atención Primaria, desde un enfoque de derechos"1 (2011), en el cual se advertía la necesidad de realizar una propuesta en territorio con la intención de trascender la capacitación teórica y centralizada en la Capital, apuntando a la intervención situada y la co-construcción de conocimientos entre universidadcomunidad. A partir de allí se realizaron propuestas que, en primer lugar, tomaron forma de proyecto de subsidio de extensión y, posteriormente, como beca de extensión, ambos de la Secretaría de Extensión de la Universidad Nacional de Córdoba. El artículo recorre las vicisitudes de una práctica en terreno con las características que le imprimen una comunidad del interior, cuya compleja trama histórica y vincular, permite y exige una constante reflexión a fin realizar una co-construcción a la hora de pensar, diseñar y abordar la demanda de intervención, poniendo a dialogar saberes y prácticas desde una perspectiva de trabajo que considera a los sujetos activos constructores de su realidad.

Palabras Claves: demanda; perspectiva crítica; prácticas extensionistas; salud; APS.

\section{RESUMO}

O presente trabalho retoma as ações e reflexões realizadas no marco de uma intervenção de extensão universitária na localidade da Serranita, Província de Córdoba, Argentina. Uma experiência sustentada ao longo de três anos que teve como origem um curso de extensão "Clínica Social: Ferramentas práticas em Atenção Primária, a partir de um enfoque de direitos" (2011), no qual se advertia a necessidade de realizar

\footnotetext{
* Professora da Universidade Nacional de Córdoba (UNC), Córdoba - Argentina. E-mail: marielcastagno@hotmail.com

** Professora da Universidade Nacional de Córdoba (UNC), Córdoba - Argentina. E-mail: mariasoldiaz@hotmail.com.ar
}

1 Responsables: Doctores Ana Aguirre y Tomás Torres Aliaga y Lic. Mariel Castagno. Equipo: Alicia Torres Aliaga, María Sol Díaz, Jesús Dib Ashur, Rosario Torres. Resolución 413/2012. 
uma proposta em território com a intenção de transcender a capacitação teórica e centralizada na Capital, apontando a intervenção situada e a reconstrução de conhecimentos entre universidade-comunidade. Diante dessa abordagem, realizaram-se propostas que, em primeiro lugar, tomaram forma de projeto de subsídio de extensão e, posteriormente, como bolsa de extensão, ambos da Secretaria de Extensão da Universidade Nacional de Córdoba. $\mathrm{O}$ artigo percorre as vicissitudes de uma prática em terreno com as características que the confere uma comunidade do interior, cujo complexo trama histórico e vincular, permite e exige uma constante reflexão a fim realizar uma reconstrução à hora de pensar, desenhar e abordar a demanda de intervenção, pondo a dialogar saberes e práticas desde uma perspectiva de trabalho com sujeitos ativos construtores de sua realidade.

Palavras-chave: demanda; perspectiva crítica; práticas extensionistas; saúde; APS.

\begin{abstract}
This paper reports actions and reflections carried out in the context of a university extension intervention in the community of Serranita, Province of Cordoba, Argentina. A Project that was developed for three years and originated in an extension course called "Social Clinic: Practical Tools in Primary Care focusing on rights" (2011), This project addressed the need to implement a proposal in the field aiming at transcending the theoretical qualification centralized in the capital city, pointing out the importance of situated intervention and reconstruction of knowledge between university and community. Considering this approach, the initial proposals comprised a Project of extension support, which later on was transformed into extension grant, both developed by the National University of Cordoba Extension Secretariat. The paper analyzes the difficulties of the field practice in an area with characteristics of a rural community, whose complex background and links, enable and require constant reflection to favor reconstruction while thinking, designing and addressing the intervention demands. It also points out the engagement of knowledge and practices in dialogue from the viewpoint of active individuals who build up their own reality.
\end{abstract}

Keywords: demand; critical perspective; extension practices; health; APS.

\title{
Los anteojos con los cuales miramos: acerca del proyecto de intervención
}

El proyecto de intervención que se analiza en el presente artículo, se llevó a cabo desde 2013 hasta el 2015, inclusive en la localidad de La Serranita, ubicada a 50km al Sur de Córdoba, Capital en la provincia de Córdoba, Argentina. El mismo se gestó a partir de un curso de extensión Universitario ofrecido por la Facultad de Psicología ${ }^{2}$. El primer ańo contó con un subsidio a proyectos de extensión ${ }^{3}$ y el segundo con beca de de la Secretaría de Extensión UNC ${ }^{4}$. Se trabajó junto con la población, con referentes de la comunidad, el centro de salud del lugar y un equipo interdisciplinario conformado por profesionales egresados (psicólogos/as, médicos/as, ingenieros/as, profesores de teatro) y estudiantes en formación de la facultad de Psicología, todos ellos con algún tipo de relación con la Universidad, como docentes o egresados de diferentes unidades académicas.

2 Curso de Extensión: Clínica Social. Herramientas Prácticas en Atención Primaria desde un enfoque de derechos. Facultad de Psicología. Universidad Nacional de Córdoba. 80 hs reloj. Resolución. Dec: 02/2012.

3 Curso de Extensión: Clínica Social. Herramientas Prácticas en Atención Primaria desde un enfoque de derechos. Facultad de Psicología. Universidad Nacional de Córdoba. 80 hs reloj. Res. Dec: 02/2012.

4 Becaria extensionista SEU-UNC: “UN ESPACIO (ambiental) PARA DOS TIEMPOS (generacionales): Un abordaje inter generacional sobre la problemática de la basura en las sierras de Córdoba". Facultad de Psicología - Facultad de Ciencias Agropecuarias. Directora: Mariel Castagno; Subdirectora: Liliana Pietrarelli; Becarias extensionistas: Ma. Sol Díaz, Lucrecia Bertoni; Equipo: Melina Rivarola, Ana Aguirre, Tomás Torres. Res. 62/2013. Universidad Nacional de Córdoba.2013.- 
El primer año de intervención constó del acompañamiento al equipo de salud en la realización de un Diagnóstico Local Participativo, este objetivo respondía a la hipótesis siguiente; los equipos de salud que contaban con iniciativas tenían poca disponibilidad y experiencia en común para dar soluciones integrales basadas en estrategias de Atención Primaria de la Salud (APS), es decir, se buscaba poner en escena la necesidad de hacer accesible la salud, como derecho de todos, garantizar universalidad, entender a la salud como parte del desarrollo local y actuar antes de que aparezca la sino entender a la salud como parte del desarrollo local. Es decir, reconocer a la población, sus miradas y prácticas.

Este diagnóstico permitió a los diferentes profesionales del dispensario "salir" del consultorio hacia la comunidad, ya que se hizo a través de encuestas domiciliarias mediante las cuales se buscaba entablar una relación con vecinos. Así se identificaron algunas problemáticas prioritarias enunciadas por los pobladores y, sobre todo, algunas respuestas a esas problemáticas que ellos mismos ya estaban pensando o poniendo en acción, pero dentro del ámbito privado, personal o familiar.

Esto permitió, por un lado, la posibilidad de que el equipo de Salud se viera a sí mismo en su proceso de constitución, espacios cotidianos y un acercamiento a la comunidad en sus diferentes ámbitos, para juntos problematizar y visibilizar posibles soluciones enmarcadas en proyectos de intervención desde un enfoque de derecho de la Atención Primaria de la Salud (APS).

Al comenzar a realizar las visitas domiciliarias para realizar las encuestas sociosanitarias los y las vecinos/as hicieron referencia al proyecto que se estaba llevando a cabo por parte del INTI (Instituto Nacional de Tecnología Industrial) ${ }^{5}$ en la comunidad. Dicho proyecto buscaba determinar el volumen de los residuos orgánicos y el tratamiento que le dispensaban los/as vecinos para luego promover y desarrollar un modelo de gestión de residuos sólidos orgánicos domiciliarios, utilizando la técnica del compostaje. Y, en un segundo momento, buscaba evaluar la calidad de ese compost para finalmente plasmar la experiencia en un Manual de Gestión que fuera útil para los municipios y comunas, favoreciendo la réplica de la propuesta ${ }^{6}$.

Tomando conocimiento de esta propuesta e identificando que el tema de los residuos era una de las problemáticas puntualizadas por los/as vecinos/as se decidió articular con el grupo responsable para pensar aportes y potenciales trabajos en conjunto. Luego de los primeros contactos y si bien los objetivos del INTI apuntaban a cuestiones técnicas, en el transcurso del proceso se identifica, al re pensar las intervenciones, que los procesos vinculares subjetivos estaban siendo remarcados por los distintos actores de la comunidad. Es así como se constata que, por un lado, que la comunicación y las relaciones establecidas en el proyecto del INTI fueron de tipo unidireccional y radial (Figura $\mathrm{n}^{\mathrm{o}} 1$ ), es decir los técnicos establecieron vínculos positivos con cada uno de los participantes que posibilitaron sostener la propuesta, sentirse protagonistas y mitigar el aburrimiento y la soledad de algunos vecinos. Que así opinaban al respecto: "Mi casa era el final del recorrido de ellos, venían a comer acá"; "Las chicas del INTI nos daban el material informativo, luego nos visitaban y nos analizaban el compost."; "Somos como conocidos con la gente del INTI, tenemos confianza, les decimos; "pase nomás".

5 Para acceder a los gráficos dirigirse al siguiente link: http://www.inti.gob.ar/compostajedomiciliario/piloto. htm

6 Evaluación del compostaje domiciliario como modelo de gestión de los residuos orgánicos. Caso piloto en la comuna Villa La Serranita en la provincia de Córdoba. Violeta Silbert, Guillermo Garrido, María Fernanda Suárez, Alejandro Benítez, Eugenio Pettigrani. Disponible en: www.inti.gob.ar/compostajedomiciliario/ 2014 
Figura 1

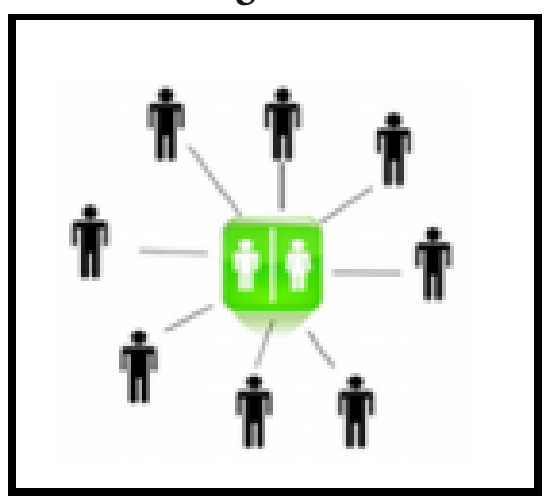

Y, por otro lado, en el proceso emergieron demandas de los vecinos, respecto a que existan procesos de implicación en las problemáticas locales que favorezcan vínculos en red, y para ello se hacían necesarias comunicaciones posibilitadoras de relaciones que contacten diferentes sujetos, instituciones y grupos de manera sostenida. (Figura $\mathrm{n}^{\circ} 2$ ).

Figura 2
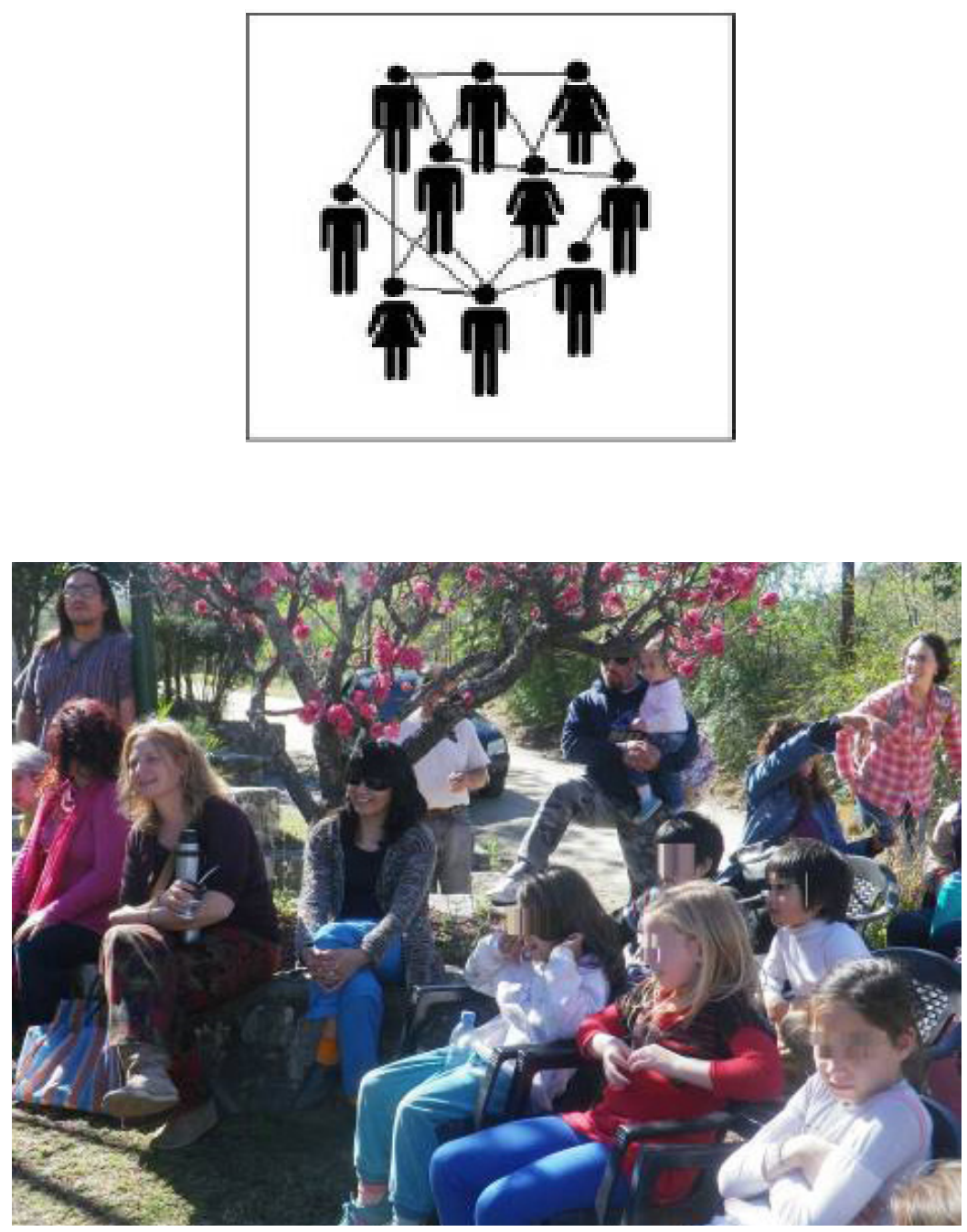

Eco Picnic en familia.

Trabajo con niños, ninas y adultos.

Visita de los cuenta cuentos. 
A partir de esas experiencias se retomaron las demandas de la población y primeras iniciativas, dando forma al proyecto de beca de extensión "UN ESPACIO (ambiental) PARA DOS TIEMPOS (generacionales)", a partir del cual se propuso hacer foco en las problemáticas prioritarias identificadas; por un lado la basura y, por otro, el aburrimiento en los adultos mayores. Para ello el proyecto apuntó a propiciar espacios de encuentro entre niños y adultos mayores favoreciendo la socialización y la réplica, de la experiencia de compostaje. Con la intención de posibilitar la transmisión oral intergeneracionalmente y con dispositivos que contemplaron lo lúdico, lo cultural y lo recreativo; dando lugar al intercambio y aprendizajes en el vínculo intergeneracional logrado entre los diversos grupos etarios. Paralelamente se buscó favorecer la configuración de promotores ambientales, desde una perspectiva de APS, buscando así abordar de manera intersectorial, interdisciplinaria y participativa las problemáticas más sentidas por la comunidad, desde su perspectiva, sus propios recursos y experiencias de articulación (CASTAGNO, et al, 2014).

\section{Reflexiones en torno al sentido y particularidad de la Extensión Universitaria}

La Universidad Nacional de Córdoba, como universidad pública, se asienta en tres pilares fundamentales: docencia, investigación y extensión. Entre ellos existe una relación de la cual se desprende la formación y prácticas profesionales de sus estudiantes, docentes y egresados, con el objetivo de alcanzar un perfil de egresado comprometido con la sociedad a la que pertenece.

Como bien lo define la Secretaría de Extensión, coordinadora de las acciones y prácticas extensionistas: "En la Universidad, la relación con la sociedad es cultivada y desarrollada principalmente a través de la función extensionista, entendida como punto articulador tanto con docencia como con investigación con capacidad de generar una relación transformadora de la realidad. Es decir que, desde la UNC, se concibe a la extensión universitaria como un diálogo de saberes que permite la construcción de un proceso de encuentro, escucha, debate, conflicto y concertación con otros actores de la esfera pública y privada, partiendo desde enfoques que privilegian la integralidad y la participación en los problemas y temas y en la definición de las acciones."

En relación con ello, el equipo atravesó procesos de reflexión a partir de los cuales se pudieran acordar puntos de partida para encarnar esta función universitaria, desde un grupo heterogéneo y diverso, tanto en los profesionales que lo componían, los vecinos, sus historias de vida, las distintas inscripciones institucionales.

En esta discusión pudimos diferenciar dos tipos de prácticas que pueden ponerse en juego en terreno: por un lado una de tipo asistencialista que intenta llegar a las comunidades pero desde una posición de saber/ poder, y por otro lado, prácticas extensionistas que buscan un trabajo interdisciplinar e interinstitucional, a través de procesos horizontales de intercambio de conocimientos producidos a niveles académicos y aquellos elaborados por los vecinos/actores sociales de la comunidad. Esta segunda modalidad tiene en cuenta tanto el conocimiento del lugar donde viven, de las necesidades que plantean y la

7 UNC, P. d. (18 de abril de 2016). www.unc.edu.ar/extension/seu/autoridades. Disponible en http://www.unc. edu.ar/extension/seu/autoridades 
posibilidad de encuentro con otros para, de esta forma, elaborar las acciones a realizar en conjunto.

La distinción a la que nos referimos no es ingenua sino que, por el contrario, responde a formas de intervenir que están en el seno de una sociedad donde la modalidad asistencialista es la más común, o la que más se vivencia en los territorios, y sobre todo al tratarse de psicólogos, porque es la intervención para la cual se está más formado y la que más esperan (y por tanto también demandan) las poblaciones de nosotros (los psicólogos): diagnosticar, intervenir, curar o "solucionar" el problema.

\section{Huellas de las modificaciones del Estado en las intervenciones en salud: Algunos impactos en esta experiencia extensionista}

Como se sabe a partir de la década de los años 90, la estructura del Estado argentino sufrió una profunda modificación, sostenida por la afirmación de su ineficiencia y de la ausencia de respuestas ante las demandas de la población. Entre dichas transformaciones, el sector de las políticas sociales fue uno de los principales afectados. La crisis económica así como las medidas de ajuste estructural, deterioraron la situación laboral en nuestro país, aumentando la tasa de desempleo, provocando caída en los salarios, incrementando la precarización laboral, con la consecuente crisis en la organización sindical y negociación colectiva.

Pese a esto, y frente al progresivo agravamiento de la situación socioeconómica, muchos argentinos llegaron a organizarse en movilizaciones de protesta, ante lo cual el Estado respondió con la distribución de planes sociales y de asistencia, principalmente alimentaria, de manera focalizada. De esta forma, la práctica universalista propia del Estado de bienestar, el cual se erigía en cierto momento como garante pleno del "bienestar" de la población, en la cobertura de bienes y servicios indispensables, era reemplazada por una mirada particularista de la acción social del Estado, dirigida ahora a la cobertura de necesidades básicas insatisfechas de determinados grupos de la población. Donde su cliente pasa a ser el pobre por desocupación.

Emilio Tenti Fanfani (1991) plantea que la idea que subyace a esta "tradición asistencialista" es que la pobreza no existe sino que únicamente existen pobres. El estado distingue así, a los más débiles, de aquellos que estarían en condiciones de realizar sus intereses y procurarse el bienestar vía el mercado. Las políticas sociales, que se dirigen a grupos vulnerables, estarían orientadas al control social, asumiendo un modelo estático de la pobreza, despolitizada; incrementando así la individualidad y acelerando procesos diversos de fragmentación y descolectivización. No solo no producen inserción laboral, sino que además sostienen la desigualdad social.

Esta manera de gestionar las políticas sociales reside en una particular concepción sobre la relación Estado, familia y mercado. Durante la vigencia de estados que se posicionan en la perspectiva de derechos, se reconoce la ciudadanía social y al mismo estado como garante debiendo velar por la universalización e integralidad. En cambio, el modelo de organización neoliberal, al que Latinoamérica y Argentina en particular están regresando, plantea que cada persona por su propia cuenta y esfuerzo deberá conseguirse el bienestar mínimo, es decir que este es concebido como un individuo autónomo y autorregulado. Sostener que la solución de los problemas sociales, consecuencias de 
estructura y organización social, están en manos del individuo, no hace más que correr la responsabilidad de lo público a lo privado. (PETRAS, 1997).

En este mismo sentido, Lo Vuolo (1999) plantea que el modo de regulación de la pobreza está influido por las llamadas visiones "tecno-liberal" y "caritativa". La primera alega que la solución del problema llegará en el largo plazo como resultado del crecimiento económico, del empleo y los micro-emprendimientos que revalorizan los activos económicos de los pobres. La visión caritativa valoriza principalmente la solidaridad moral "como ética personal de los donantes" y promueve la fraternidad entre los propios pobres, alentándolos en su auto-organización económica. Esta visión reconoce el contenido económico del problema, pero descarta que la solución venga por una modificación de la política económica. Por lo tanto, en este tipo de programas, se trata de identificar beneficiarios es decir, a quienes "merecen" mayor asistencia.

Aunque en la década que finaliza junto al ańo 2015, nos encontramos con un Estado que incorporó un discurso de apertura a un rol más activo en la vida de la población en términos de ampliación de sus políticas pero, a pesar de un importante impacto de universalismo de los derechos sociales, en el sector salud se evidencia que el Estado continuó sin lograr este propósito, ya que varias de las políticas sanitarias fueron focalizadas, dirigidas a sectores empobrecidos o de riesgo. Cuestión que, además, se profundizó durante este año con en cambio de gobierno nacional.

Como advierte Zaldúa "La paradoja es que ante las inequidades y desigualdades sociales, se desarrollan reformas de políticas públicas basadas en la mercantilización de los servicios sanitarios y una supuesta ineficiencia financiera y técnica que produce, una doble exclusión, una doble vulneración y abandono. Las consecuencias de la fragmentación y selectividad social evidencian la falta de universalidad en el derecho a la salud" (ZALDÜA, 2010, p. 11).

Las políticas de salud han estado siempre marcadas por intereses mercantilistas, corporativistas, económicos y políticos. Relegando al estado el papel de interventor solo en las poblaciones marginales, en prevención y asistencia médica no rentable. Esto produce que nunca se llegue a una cobertura total, potenciando la estratificación del sistema frente a los principios de universalismo y ciudadanía, así como la inequidad en el acceso a los servicios. Como dicen Torres y Torres (2010), el sector público de la salud se encarga de las personas en situación de pobreza y el sector privado de los sectores pudientes. La salud deja de verse como un derecho y es tratada como un bien de consumo. Mientras que la atención especializada y compleja de la salud pertenece a la esfera de lo privado y se convierte en un derecho inaccesible para los sectores en situación de pobreza. Además, desde esta concepción, le es relegada la prevención al sector público, lo cual impide superar la dicotomía entre medicina preventiva y curativa.

En el caso de nuestra provincia, Córdoba, estas marcas del contexto se plasman en el proceso de descentralización de los servicios de salud.

En el caso de nuestra provincia, Córdoba, estas marcas del contexto se plasman en el proceso de descentralización de los servicios de salud. Dicha descentralización de servicios sociales, pero particularmente los de salud, comienza a formar parte de la agenda del estado provincial a partir de 1987, cuando se realiza la reforma de la constitución provincial. Sin embargo, se concreta efectivamente a finales del año 95, con la crisis de financiamiento de la provincia. Para saldar esta crisis, el Estado toma medidas de ajuste fiscal para mejor las cuentas públicas a corto plazo. En este contexto se produce la transferencia masiva de servicios sanitarios de baja complejidad, desde la jurisdicción provincial a la municipal (ASE, 
2006). "Se establece que la provincia avanzaría en el traspaso a nivel municipal, de todos los efectores calificados como de baja complejidad, guardándose para sí los de complejidad

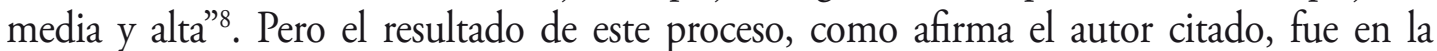
mayoría de los casos una pérdida significativa en la calidad de los servicios, acentuando la heterogeneidad en las respuestas sanitarias de cada jurisdicción, profundizando las desigualdades regionales y provocando grandes costos a la eficiencia y equidad.

Asimismo, no ha sido posible ni a nivel provincial ni a nivel regional, consensuar un acuerdo que establezca para cada uno de los actores los márgenes dentro de los cuales debe prestarse los servicios de salud a su cargo. Esto lleva a que las características que asume cada servicio de salud municipal dependan de la decisión política del comunal o intendente municipal de turno. Incluso la idea de que se deban limitar a la atención primaria y al primer nivel de atención queda sujeta a diversas interpretaciones en cuanto al tipo de servicio y cantidad de especialidades que considere. La política de salud entonces adquiere un elevado nivel de discrecionalidad.

Así y en la mayoría de los casos, el trabajo en los municipios se centra en la demanda espontánea, con la figura del médico como eje del servicio y escaso o nulo trabajo comunitario. La prevención y promoción de la salud se limita a algunas acciones en escuelas o difusión de información. En este sentido, una vez más queda evidenciado que el modelo médico hegemónico sigue impregnado en las prácticas actuales en salud. Y además se ha transformado en el "sentido común" en cuanto a lo considerado como "buena medicina". Lo mas "confiado" y "valorado" por las comunidades serían las tecnologías de última generación y los especialistas (ASE I., 2006).

\section{Del modelo médico hegemónico a la salud colectiva}

Las prácticas médicas asistenciales continúan influenciadas por el modelo médico hegemónico, donde prima el diagnóstico y tratamiento individual. Las principales características estructurales del mismo son: biologicísmo, individualismo, a historicidad, a-sociabilidad, mercantilismo y eficacia pragmática, si bien dichos rasgos pueden observarse en la medicina practicada antes del siglo XIX, durante este siglo se profundizaron, convirtiéndose en las características dominantes de la biomedicina. El biologicísmo y evolucionismo que caracterizaron a este modelo, excluyeron la lectura de los procesos históricos y los determinantes socio-económicos, culturales e ideológicas respecto a la causalidad y desarrollo de los padecimientos. (MENÉNDEZ, 2005). La superioridad de la biomedicina se basa en su supuesta mayor eficacia comparada con los otros saberes populares y académicos.

Por otra parte, no solo el discurso del orden, sino también los imaginarios sociales confluyen para sostener este modelo sin modificaciones. Más de una vez en nuestras intervenciones los pobladores han igualado la intervención del médico a prácticas de salud, dicho deslizamiento de sentido es parte de un proceso de mistificación, como dice Ana Fernández (1989). Sumado a esto, la consideración de la salud y la enfermedad como bienes con valor de cambio, concepción que incide en la forma que adquieren las prácticas, relegando cada vez más la APS.

8 Ase, I. La descentralización de servicios de salud en Córdoba (Argentina): entre la confianza democrática y el desencanto Neoliberal. Salud Colectiva. 2(2): 202. 2006. 
Ante la presencia de un modelo que se agota y no tiene respuestas para las poblaciones más grandes que son los pobres, podemos mencionar como alternativa los presupuestos conceptuales de la salud colectiva sobre el Proceso de Salud-EnfermedadCuidado (PSEC), que nos permiten una forma distinta de abordar las problemáticas de salud y que retomamos como esquema referencial y conceptual en nuestra práctica:

"Pensar al PSEC como construcciones socio históricas de procesos complejos y macroestructurales, que no se limitan a lo médico y que incluyen los diferentes determinantes sociales en los cuales viven las poblaciones. Dejando de lado la supremacía de los reduccionismo causales. Cuyos procesos son construcciones colectivas e integrales en contraposición a procesos individuales y preventivos. Incluye procesos creativos y saludables en lugar construcciones negativas de la salud focalizadas en la enfermedad y lo negativo. Reconoce el aporte que pueden realizar los múltiples actores y formas de saberes locales y populares, pensando en la salud desde la perspectiva de diversas culturas. Deslegitimando la visión euro céntrica y unicultural, en contra de responsabilidades sólo institucionales o desde saberes disciplinares y profesionales. Procesos donde lo fundamental es una praxis participativa, en relaciones simétricas y con prioridad de valores de ciudadanía, equidad y derechos, en contra de interés en conocimientos meramente académicos e intervenciones desde la asistencia o tutela". (TORRES y TORRES, 2010, p. 22).

Sostenemos que este trabajo de reflexión, así como en la intervención realizada que le dio lugar al mismo, parte de una concepción de salud como construcción histórica y social. La misma constituye una síntesis de calidad y condiciones de vida de las personas así como de sus grupos de pertenencia, atravesada a su vez por cuestiones económicas, culturales y políticas. "La salud y enfermedad no son entidades naturales, universales, sino construcciones sociales, que sólo existen como tales, en el marco histórico-cultural en que se constituyen como entidad: lo que es sano para una sociedad no lo es necesariamente para otros" (TORRES y TORRES, 2010).

Los enfoques del movimiento de Salud Colectiva ${ }^{9}$ latinoamericana confrontan al modelo médico hegemónico en el campo científico, político y en las prácticas. Dicho movimiento, para Fleury (2007) pone en evidencia la crisis de la medicina mercantilizada, como también de su ineficiencia, en cuanto a la organización de un sistema de salud por no responder a las demandas predominantes, organizado de forma democrática en su gestión y administrado en base a la racionalidad en la planificación". (FLEURY. S., 2007, p. 150). Como sostienen Zaldúa y Bottinelli (2010) "Los procesos determinantes y mediadores de la desigualdad e inequidad se expresan en los procesos salud-enfermedadatención- cuidado en la distribución poblacional, en los grupos sociales, en las diferencias de género, de etnias"10.

De alguna forma podemos conjeturar que la práctica generada a partir de esta experiencia pensada desde la PSEC, gestionada desde agentes estatales en calidad de extensionistas y accionada a través del diálogo y la participación comunitaria, se comenzó a instalar cada vez con más fuerza en las políticas de gobierno comunal de la Serranita. Así, el abordaje comunitario de la problemática de la basura, la constitución de un grupo de defensa civil para los incendios y hasta la organización de un centro de jubilados

9 Salud Colectiva, es un movimiento sanitario brasileño incorporado para denominar lo que hasta entonces se conocía como "Salud Pública".

10 Zaldúa.G, y Bottinelli.M. Praxis Social comunitaria en salud. Campos epistémicos y prácticas participativas. Buenos Aires. P.10 .2010. 
para contrarestar el aburrimiento y la soledad de los adultos mayores de la comunidad, comenzaron a formar parte de la agenda de la gestión de la Serranita.

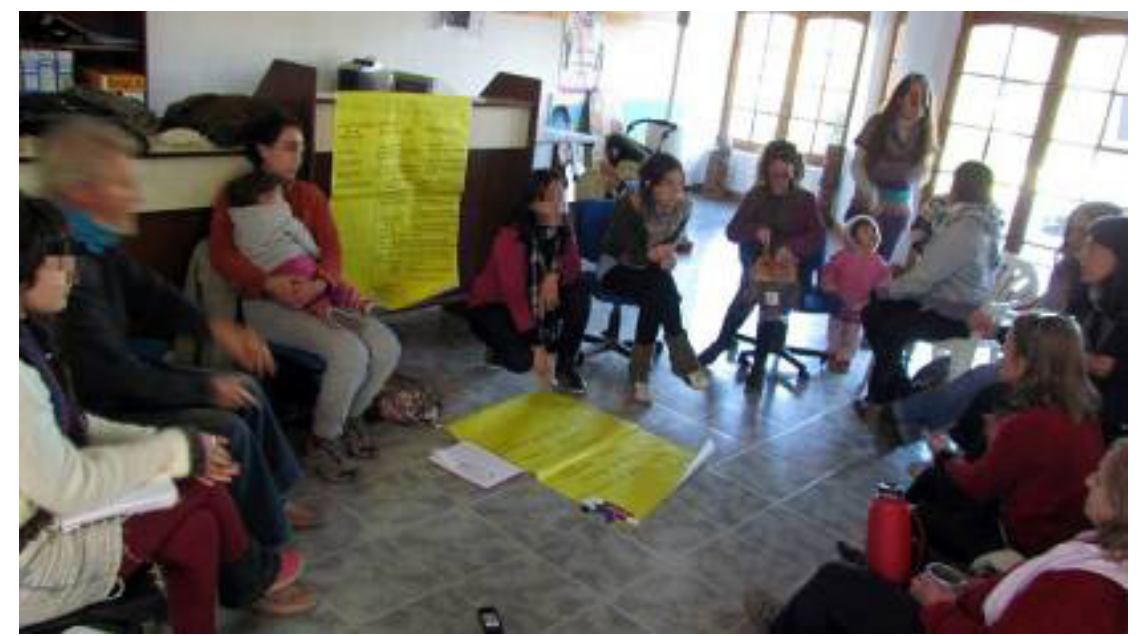

Ecopicnic. Reunión con vecinos en la comuna donde se trabaja la preocupación por las consecuencias sobre los últimos incendios. De la misma se pone fecha para la próxima reunión donde se trataría la conformación de Defensa Civil.

\section{Construcción de la demanda en terreno desde una perspectiva situada e histórica}

En orden a realizar una lectura de la demanda y una construcción en conjunto con la población del objeto de intervención, se trabajó considerando a los vecinos como actores y bajo una constante elucidación crítica donde analizamos también a nosotros mismos interviniendo allí. En este sentido, y entendiendo que este ya no se define (no puede definirse) por una única referencia a un grupo social que le confiera un espacio de orden estable, no podemos negar que los "procesos identitarios como movimientos psicosociales dinámicos más que en una identidad social objetiva inmutable"11 y entendiendo al sujeto social como un ser complejo donde hay una articulación constante entre lo psíquico, lo social, lo simbólico, lo cultural, éste va creando múltiples pertenencias y se va constituyendo a través de multireferencias. Este "movimiento identitario" debemos tener en cuenta para que las intervenciones no queden desancladas o impuestas.

Al diseñar cada intervención fue (y sigue siendo) importante pensar y de-construir los aspectos sobre "lo dado" en los discursos y prácticas que los actores producen. No solo para desmitificar el cotidiano junto a los actores implicados, sino también para sostener la relevancia de los mismos como los primeros y principales capacitados para significar los hechos sociales. Nos referimos a la doble hermenéutica que en prácticas extensionistas implica mantener una constante actitud de escucha y de "humildad epistemológica" (ARAUJO, 2011).

Bózzolo (2000) nos advierte que si encontramos lo que esperamos, nada nuevo se produce sino que sólo se constata lo ya sabido. Pero si, en cambio, no podemos explicar lo que ocurre allí tenemos un resquicio donde marcar una diferencia. En relación a ello tuvimos la posibilidad de preguntarnos ¡en qué medida las propuestas que se presentan como proyectos de extensión van de la mano con las necesidades actuales de la comunidad?. Justamente a partir de las experiencias vividas el equipo pudo constatar que, aún, habiendo pensado una propuesta en base a un diagnóstico

11 Araujo, Ana María De historia e historicidad. De distancias ocultas...de nuestras identidades en Habitus psico-socio-cultural de la sociedad motevideana. Montevideo. Ed. ARGOS. 1996. 
local participativo, lo que emergía para ese colectivo de vecinos con el que se trabajaba en ese momento en particular, no tenía que ver con la "preocupación por el ambiente", lo cual parecía una obviedad por el trabajo con las composteras, sino que, la escucha nos permitió acceder a lo que había generado los incendios en esta comunidad: una experiencia traumática, que habían sufrido los pobladores en el año anterior y temían volver a sufrir. Dada la insistencia del relato de la vivencia con semejante tonalidad afectiva, nos interpeló la práctica y complejizó la demanda.

Entonces nuevamente advino la pregunta ¿Qué esperan de nosotros? ¿Qué ofrece sin decir nuestra inscripción profesional como psicólogos? ¿Cuál es el lugar de la demanda en las prácticas extensionistas? Incluso cuando, a partir de las características de las convocatorias a becas y subsidios de financiación, puede perderse como horizonte esta relación inherente a la extensión: que la intervención se construirá con la población. No a priori, no sin su conocimiento y efectiva vinculación, no sin haber logrado un encuentro genuino entre vecinos y académicos.

Muchas veces el proceso de lectura de "lo que pasa" se ve afectado por el cumplimiento de los aspectos formales, los tiempos, el plan de trabajo y, hasta los recursos asignados, pero estas presentaciones no pueden ser consideradas desconectada del diálogo con la comunidad. Si bien de alguna forma el objeto de intervención se define ya en la escritura de la propuesta debemos estar atentos a las transformaciones del mismo, a la interpelación que plantea el contacto sucesivo con la comunidad.

Implica ir delineando un objeto de intervención a partir de la tensión entre la demanda de los pobladores y la posición del equipo técnico (BONVILLANI, 2005). Distinguir "encargo" de "demanda", para pensar desde donde proviene la intencionalidad de la intervención y diferenciar si busca una conservación del statu quo o la significación subjetiva desde otro registro, otra lectura, no hegemónica ni homogenizante, de las supuestas "necesidades". Análisis que debe ser tenido en cuenta para marcar la diferencia entre abonar procesos de transformación o perpetuar las relaciones de poder, en la dialéctica instituido-instituyente.

En este sentido, durante la experiencia ejercitamos una revisión crítica de nuestras intervenciones, atentos a no imponer o cerrar sentidos, por el contrario, tratamos de poner a disposición condiciones para el diálogo que permitieran la emergencia de nuevas significaciones. Saber que en esa enunciación de algo, ese algo es el "borde" de la demanda, y para poder desconstruirla debemos estar atentos a no reproducir modos de estar, hacer, pensar, desde posiciones dominantes. Y por supuesto que, dentro de estas posiciones, están las lógicas universitarias con sus requisitos, valoraciones, indicadores y expectativas de resultado.

Creemos que en el proceso fue positivo empezar analizando nuestras implicanciones, como nos afecta la experiencia, que nos pasa, y estar atento el bagaje de conceptos teóricos, técnicos, que pudieran llegar a encasillar un fenómeno, que inhiba la apertura a diálogos, otros actores, otras realidades. Pedrosian (2003) propone estar en silencio fomentando que el otro se exprese y que los que constituyan el fenómeno nos cuenten. Mantener la tensión entre distanciamiento y proximidad para darle lugar al otro desde la escucha y la consideración del proceso como transcurrir, como camino y transformación.

Nuestros aportes de saberes académicos, técnicos, de experiencias o experticias se ponen en juego en las intervenciones que realizamos, pudiendo utilizar diferentes herramientas que permiten alcanzar los objetivos planteados para cada etapa, pero la definición de cómo será ese dispositivo (o sea sus especificidades) requerirá de revisarlo desde sus dimensiones ontológicas, epistemológicas, metodológicas, éticos y políticas. 
También fue necesario ir revisando nuestros dispositivos, e ir reinventándonos como equipo, ya que estos van perdiendo eficacia o se agotan. Es un desafío para poder sostener el trabajo a lo largo de los tres años consecutivos en la Serranita renovar las herramientas de las intervenciones. Principalmente para dar respuestas en un espacio social en cuya trama compleja los actores toman diversas posturas en relación a su pertenencia al lugar y las prioridades que van identificando. Fue necesario entonces entre el equipo, los pobladores y los profesionales, ir identificando los puntos de fuga o las oportunidades de creación que permita abrir espacios de subjetivación, en dispositivos como: los espacios de prevención del fuego, los eco-picnis, las jornadas de "Cuentos y semillas", el recorrido de identificación de flora y fauna y, finalmente, el taller de germinación.

Para ello fue muy importante, en la práctica extensionista, tomar una postura flexible y dar lugar a la complejidad, poniendo en juego los saberes que tanto vecinos como equipo poseen para construir una base comunicativa en común, que exigía la coordinación de actividades incorporando herramientas locales existentes, ejercitando una escucha atenta, amoldando objetivos. Esta fue la forma que adquirió el encuentro entre universidad y comunidad con el desafío de sensibilizar las instituciones políticas, para movilizar voluntades, financiación, apoyo y sostenimiento a la hora de configurar los abordajes a las demandas.

Retomando a Foucault, Carlos Szulkin (2003) define al dispositivo como "modalidad específica de intervención en función de categorías teórico técnicas elegidas que se dirimen en el campo de aplicación y en consecuencia es una virtualidad específica de la cual se esperan determinados efectos." (FOUCAULT en Szulkin, 2003).

Un aporte central de esta experiencia fue la construcción de dispositivo al que denominamos "Eco-picnis", los cuales se llevaron a cabo para dar consistencia e identidad a los espacios de encuentro entre vecinos de tan diferentes inscripciones identitarias. Los mismos sirvieron para compartir experiencias en relación al ambiente que habitamos. Así, a lo largo de un año de se realizaron ocho "eco-picnics" en diferentes lugares geográficos de localidad, tales como el edificio comunal, la Cooperativa eléctrica, el Centro de Jubilados, casas particulares de vecinos, el balneario, la canchita de Fútbol y la placita.

Los eco-picnis, que iniciaron con el propósito de construir y socializar prácticas de compostaje, se transformaron en un espacio de diálogo y escucha para abordar el cuidado del ambiente, con un sentido reparador y, psíquicamente, elaborativo en relación con el miedo al fuego y las consecuencias de la experiencia traumática de los incendios vividos por los pobladores. A partir de estos espacios de encuentro, se fueron generando lazos en los cuales se pusieron en juego la solidaridad y el compromiso a la hora de pensar en problemas comunes. A la vez que, atendiendo a la necesidad colectiva de espacios de hacer frente a la soledad y al aburrimiento, se fueron tornando momentos recreativos con propuestas de cuenta cuentos, taller de germinación y de arte, tanto para adultos como para niños.

Esto último fue posible a través del trabajo sostenido con los niños de la escuela primaria y el jardín de infantes, por parte del equipo extensionista en el primer año, a través de la articulación con el proyecto de salud bucal que apoyamos mediante el aporte artístico, con títeres y canciones, que renovó el dispositivo que se venía implementando desde el centro de salud. A su vez, se integró este tema, con el reciclado de basura, produciendo porta-cepillos de dientes con botellas de plástico en desuso para colgar en el comedor de la escuela a fin de ser utilizados después de cada almuerzo. 


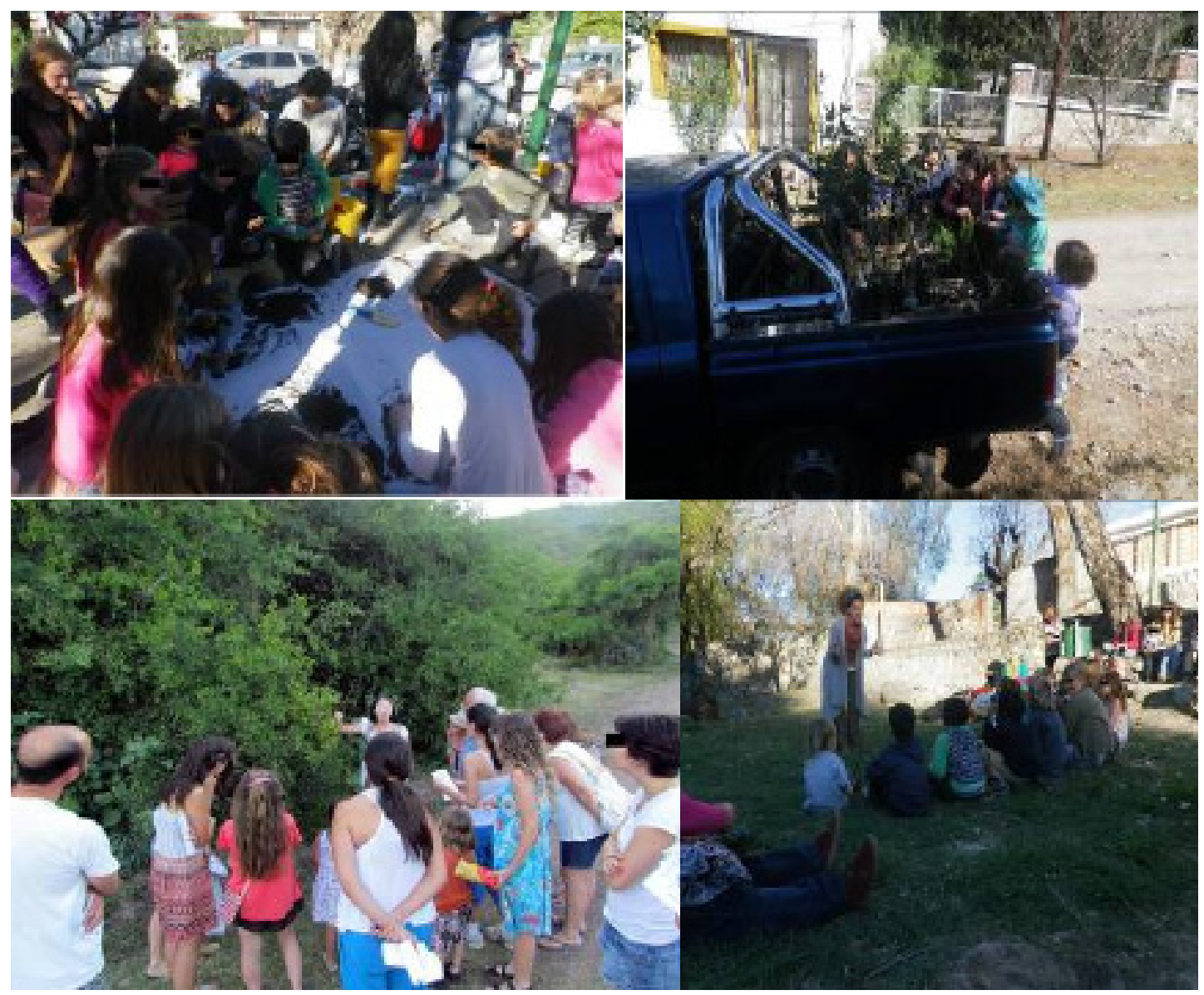

De arriba abajo, de izquierda a derecha: Foto 1: eco picnic -taller de germinación con niños/as y familia. Foto 2: árboles nativos para reforestar donados por una comunidad vecina. Foto 3: recorrido de reconocimiento de flora autóctona, coordinado por una vecina de la comunidad, bióloga. Foto 4: eco picnic -encuentro de cuenta cuentos. Cuidando el ambiente desde los cuentos y relatos.

Dentro de los logros, es importante destacar también, que a partir del diagnóstico local participativo que se acompañó desde el proyecto, fueron retomados los datos y resultados por parte de los miembros del equipo de salud, en el marco de un trabajo interdisciplinario, para proponer y acompañar la creación del Centro de Jubilados que desde hace un año, se constituyó en la Serranita.

\section{La extensión universitaria, una forma de construir lo común}

Según Castoriadis (1998), elucidar es pensar lo que se hace y saber lo que se piensa. Desde donde partimos y en relación al devenir de la intervención, reflexionar en equipo sobre las diferentes acciones buscando visibilizar que aporte realizado y también qué obstaculizó cada proceso, nos permitió elucidar críticamente nuestras prácticas extensionistas.

Retomando aportes del Análisis Institucional podemos afirmar que toda idea, invención, cuando toma una forma social entra en institucionalización y por tanto pierde su radicalidad. El dispositivo de análisis tendrá que ver con confrontar lo instituido y lo instituyente, observar y analizar los acontecimientos que surgen, cuando lo no dicho emerge y se expresa. En ésta experiencia extensionista lo emergente fue la repetición 
del relato de los incendios, que aparecía como forma de enunciar al menos dos situaciones; por un lado, la vulnerabilidad ante el fuego propiamente dicho y la escasez de herramientas materiales, organizativas, vinculares y simbólicas para hacerle frente; y por otro, propiciaba un proceso de exigibilidad al estado en su nivel municipal y a sus referentes, para evitar estas situaciones.

Surge como emergente junto al momento político y social (procesos pre eleccionarios) que se estaba viviendo, por lo que tanto el espacio como las respuestas posibles fueron disputadas por las instituciones de la comunidad, así como también por un grupo de pobladores que se denominaron "vecinos autoconvocados".

Cuando se postula la caída de la institución que nos contuvo históricamente, se hace referencia a la fuerza con la que se instaura en nuestra subjetividad la misma: el Estado, la confianza en las autoridades, en su protección, en la estabilidad. Ciertas formas de definir situaciones y prácticas como "las adecuadas" aún cuando no comprendamos porqué lo son, hacen referencia a la subjetividad instituida. Pero a lo que nos afrontamos, en esta instancia de la experiencia, es a la subjetivación. Como proceso colectivo donde se va más allá de esa subjetividad instituida, que es lo que permite desarticular o criticar lo instituido en un primer momento. (LEWKOWICZ I., 2004). Como dice el autor citado, la idea de ciudadano regido por una ley que es igual para todos, educado por un estado al que le delega su poder, se enfrenta ahora a un estado que se enuncia incapaz de dar soluciones. En este marco trabajamos, receptando esa queja de inacción, omisión o negación de la solución de las necesidades de las comunidades. Se presenta así un desafío, respecto a cómo desandar esa queja y transformarla en acción, cómo replantear la solución en el seno de la comunidad y a través de sus herramientas y potencialidades.

La intervención favoreció, luego de intensos procesos de debates, conocimientos producidos y necesidades explicitadas por los vecinos respecto al problema del fuego, que sean abordados por la conducción de la comuna, en articulación con otras instituciones como la cooperativa eléctrica, para la organización de espacios de capacitación de Defensa Civil. Estos resultados indican la fuerza que adquiere el trabajo conjunto cuando es visibilizado mediante acciones contundentes, y como también redunda en políticas locales y viceversa.

Guatari (1998), sostiene que “(...) la cultura de masas produce individuos: individuos normalizados, articulados unos con otros según sistemas jerárquicos, sistemas de valores, sistemas de sumisión; no se trata de sistemas de sumisión visibles y explícitos, como en la etología animal, o como en las sociedades arcaicas o precapitalistas, sino de sistemas de sumisión mucho más disimulados". Pero aún así "hay (...), en la relación consigo, en la producción de subjetividad, la posibilidad de ejecutar el acontecimiento; existe la posibilidad de sustraerse a la producción serializada y estandarizada de la subjetividad." Pero esta posibilidad ha de ser construida, las posibilidades han de ser "creadas" y desde nuestras vivencias, experimentadas. ¿Es entonces esa la función de los equipos extensionistas interventores en salud? Como dice Enríquez (2005) posibilitar la creación de un imaginario motor, común, convocante. El mismo autor plantea que los equipos de intervención prestan especial atención a las significaciones que la sujetos hacen de sus propias acciones y los acompañan a buscar otras, no como expertos, sino como alguien que reconoce en el proceso todo tipo de saberes. Posibilitar el desplegar del sujeto, aumentar (o tratar de aumentar) la posibilidad de actuar de los sujetos- 
actores no sólo de manera más intensa sino siendo consciente de ello (ENRÍQUEZ, 2005).

Sin embargo, no se puede dejar de mencionar que el trabajo extensionista depende en su mayor parte de la voluntad, responsabilidad y compromiso de cada equipo en un marco tanto material como simbólico adverso hacia esta función universitaria. Por lo que la lucha es doble: Por un lado, para dar visibilidad de estas actividades a otros estudiantes y egresados a fin de ampliar la comprensión de la importancia de la multiplicación de las experiencias y, por otro, hacia las instituciones encargadas de destinar los recursos materiales y humanos, para reforzar el apoyo a al trabajo extensionista mancomunado y, muchas veces, invisibilizado y deslegitimado comparativamente a la jerarquía académica de la investigación (y de cierta investigación por sobre otras).

Finalmente resta decir, que consideramos que el trabajo sostenido por casi cuatro años, promovió la adquisición de herramientas para el abordaje de problemáticas prioritarias desde lo grupal, lo interinstitucional y lo comunitario. Dando además una nueva institucionalidad al proceso colectivo iniciado, que hará que no se pierda con el retiro del equipo extensionista del territorio. Además, Y en continuidad con el trabajo en APS que se sigue desarrollando en la localidad, la intervención permitió un conocimiento más profundo y complejo de los componentes sociales de la salud, desde el paradigma de Salud Colectiva. Conocimiento construido desde un trabajo comunitario articulado entre los referentes sanitarios y políticos de La Serranita y la Universidad Nacional de Córdoba, que no solo impactó en políticas locales sino también en las subjetividades implicadas en dicho proceso, inclusive en las nuestras.

\section{Bibliografía}

ARAÚJO, A. M. De historia e historicidades. De distancias ocultas...de nuestras identidades. En Habitus psico-socio-cultural de la sociedad motenvideana. Montevideo. Ed ARGOS. 1996.

ARAÚJO, A. M.. Sociología Clínica, una epistemología para la acción. Montevideo. Ed. Psicolibros Universitarios. 2011.

ASE, I. La descentralización de servicios de salud en Córdoba (Argentina): entre la confianza democrática y el desencanto Neoliberal. Salud Colectiva. 2(2): 202. 2006.

BENÍTEZ, et al. Evaluación del compostaje domiciliario como modelo de gestión de los residuos orgánicos. Caso piloto en la comuna Villa La Serranita en la provincia de Córdoba. Disponible en: www.inti.gob.ar/compostajedomiciliario/ Consultado: $11 / 2016$.

BOZZOLO, R. El "grupismo" como obstáculo. Ponencia presentado en el encuentro "En torno a lo grupal" organizado por Inserción, Paraná, Agosto de 2000, y publicado en Campo Grupal" № 18, Buenos Aires, Octubre de 2000. 
BOZZOLO, R., et. al. El oficio de intervenir. Políticas de subjetivación en grupos e instituciones. Capitulo: Recorrido subjetivo de un equipo. $1^{\text {a }}$ ed. Buenos Aires. Editorial: Biblios, 2008.

CASTAGNO, M., et al. Proyecto: "Ampliar horizontes acortando distancias. Sensibilización y capacitación de equipos de Salud de las sierras del sur cordobés" Facultad de Psicología y escuela de Trabajo Social Fac de Derecho y Cs Sociales. Córdoba. 2012.

CASTAGNO, M., DÍAZ, M. S., et al. Informe Técnico Final Subsidio SEU: Ampliar horizontes acortando distancias. "Sensibilización y capacitación de equipos de Salud de las sierras del sur cordobés". Facultad de Psicología y escuela de Trabajo Social Fac de Derecho y Cs Sociales. Córdoba. Sin publicar.

CASTORIADIS, C. Imaginación, imaginario, reflexión. En Hecho y por hacer. Pensar la imaginación, Buenos Aires, Ed. Eudeba (p. 267-331).1998.

CRISTIANO, J. Lo social como institución imaginaria. Castoriadis y la teoría sociológica, Villa María, Ed. EDUVIM (completo). 2008.

CORREA, A. et al. Notas para una Psicología Social... como crítica a la vida cotidiana. Ed. Brujas. Córdoba. 2003.

CORREA, A. y PAN, M. La intervención Psicosocial: cuadernos del campo psicosocial. n 1, Ed. Brujas. Córdoba. 2005.

DÍAZ, M. S. y BERTONI, L. Beca extensionista SEU-UNC: “UN ESPACIO (ambiental) PARA DOS TIEMPOS (generacionales): Un abordaje inter generacional sobre la problemática de la basura en las sierras de Córdoba". Facultad de Psicología - Facultad de Ciencias Agropecuarias. Córdoba. 2013.

DÍAZ, M. S. y TORRES, R. Tesina de la Licenciatura en Psicología. "Prácticas comunitarias en salud que aportan a reproducir o transformar el modelo hegemónico de la institución sanitaria en la localidad de Anisacate. Facultad de Psicología. Córdoba. 2012.

ENRIQUEZ, E. De la formación y de la intervención psicosociológicas. Rev. internationale de Psychosociologie. Traducción v. 1, no 1. 1994. Traducido por Ana Correa en Cuadernos de Investigación, Intervención, Formación y Capacitación del campo psicosocial. n 1. Ed. Brujas: 2002.

ENRÍQUEZ, E. La intervención Psicosociológica: un debate sobre la Teoría y las Practicas. En La intervención psicosocial: cuaderno del campo psicosocial. Compiladores: Ana Correa y Monica Pan. 1ed. Córdoba. Ed. Brujas. 2005. GUATTARI, F. El devenir de la subjetividad. Santiago de Chile, Ed. Dolmen. 1998.

LAZZARATO, M. Del biopoder a la biopolitica. En Revista MULTITUDES. Volumen $n^{\circ} 1$. Francia: Marzo 2000. 
LAZZARATO, M. La máquina. Epilogo del libro de Gerald Raunin Algunos fragmentos sobre las máquinas. Traducción Marcelo Expósito, revisada por Joaquín Barriendos. Semiotex(e)/Intervention. 2006.

LEWKOWICZ, I. Pensar sin Estado. La subjetividad en la era de la fluidez. Ed. Paidós, Buenos Aires. 2001.

LOURAU, R. Libertad de Movimientos. Una introducción al Análisis Institucional. Ed. Eudeba. Buenos Aires. 2001.

MENÉNDEZ, E. L. De sujetos, saberes y estructuras: introducción al enfoque relacional en el estudio de la salud colectiva. $1^{\text {a }}$ ed. Buenos Aires. Ed. Lugar. 2009.

MENÉNDEZ, E.L. El modelo médico y la salud de los trabajadores. En Revista Salud Colectiva, v.1,. n 1, p.9-32. Instituto de Salud Colectiva Universidad de Lanús. Bs As. 2005.

MENÉNDEZ E. L. y SPINELLI H. Participación social ¿para qué? Ed Lugar. 2006.

PALOMAS, S. Estrategias metodológicas para la promoción de la salud comunitaria Los Títeres tienen la palabra. $1^{\circ}$ edición, Impreso en Argentina; Espacio Editorial. 2002

PEDROSIAN, A. La escucha activa en la comprensión crítica. J. Barceló (Comp.), Sociología Clínica (pp. 37-44). Montevideo, Ed. Universidad de la República. 2003.

SAFORCADA. E. Perspectiva ecológica-sistémica de la salud. Cap. 5. En Saforcada y Castella Sarriera (comp) "Enfoques conceptuales y técnicos en psicología comunitaria". BsAs Tramas Sociales. Paidós. 2008.

SPINELLI, H., et al. Equidad en salud: teoría y praxis. En Salud Colectiva" Spinelli H (comp). Ed Lugar. Buenos Aires. 2004.

TORRES, T. y TORRES, A. ¿Por qué callan si nacen gritando? Poder, accesibilidad y diferencias culturales en salud. Iruya 1978-2008. Ed. ENDEPA Fomosa. 2010. 
TORRES ALIAGA, T. y TORRES SECCHI, A. Poder accesibilidad y diferencias culturales en Salud. Iruya. 1978-2008, Cap. II, pág. 21 y 33. Ed. ENDEPA. Formosa. 2010.

ZALDÚA, G. y BOTTINELLI, M. Praxis Social comunitaria en salud. Campos epistémicos y prácticas participativas. Buenos Aires. Ed. Eudeba. 2010.

WHITEHEAD M. Los conceptos y principios de la equidad en salud. Centro de documentación. 1991. UNC, P. d. (18 de 4 de 2016). www.unc.edu.ar/extension/seu/autoridades 